\title{
Loss of vision secondary to toxic optic neuropathy due to gutka overdose:A Case Report
}

\begin{abstract}
Introduction: Toxic optic neuropathy is defined as the damage caused by a toxin to the optic nerve fibers. There is a vast list of toxins that may lead to optic neuropathies. One of the toxins among this list is tobacco. Gutka being an oral form of tobacco, however, has not been previously related to optic neuropathy.

Case presentation: We present a case of a 22 -year-old male who presented with decreased far vision and gradually increasing photophobia. He had a history of gutka usage, a form of smokeless tobacco. Investigations revealed a central scotoma on FOV, a swollen optic disc on OCT and deranged Liver function tests. A diagnosis of RIGHT + LEFT optic neuropathy secondary to a chemical toxic was made, which in this case, was gutka.
\end{abstract}

Conclusion: Consumption of gutka can lead to irreversible toxic optic neuropathy.

Keywords: toxic optic neuropathy, gutka, smokeless tobacco, vision loss
Volume II Issue 5 - 202I

\author{
Irfan Kabiruddin Jeeva, Sidra Masud, Syed \\ Hasan Raza Abidi, Tazeen Saeed Ali, Ayesha \\ Akbar Waheed, Sobia Tariq Siddiqui \\ Aga Khan University Hospital Karachi, Pakistan
}

Correspondence: Irfan Kabiruddin Jeeva, Section of

Ophthalmology, Aga Khan University Hospital, Stadium Road, Karachi, Sindh, Pakistan, Tel +92340121628I,

Emailijeeva@icloud.com

Received: August 04, 2021 | Published: September 27, 2021

\section{Introduction}

Gutka is a form of smokeless tobacco associated with oral cancer. ${ }^{1}$ Pakistan, India and Bangladesh have the highest consumption rate of gutka in the world, and according to the WHO there are approximately 10 million of Pakistani population addicted to smokeless tobacco. ${ }^{1-3}$ Tobacco is a known cause of toxic optic neuropathy. ${ }^{5}$ As gutka is a form of tobacco, one can conclude that it can cause optic neuropathy, however, existing literature does not link its consumption to the condition. We therefore report a case of toxic optic neuropathy resulting from presumed excessive consumption of gutka.

\section{Case presentation}

A 22-year-old man, with no known co-morbid, developed severe headache and blurred vision. According to him, he had consumed almost 5 times the usual amount of gutka earlier in the day. The headache gradually resolved but the visual blur continued. He was able to access medical help a month later, where he was prescribed oral prednisolone and investigated with MRI that was reported as normal. Two month later he presented to eye clinic at the Aga Khan University Hospital, Karachi, Pakistan. On examination, the patient's best-corrected visual acuity was counting fingers at 2 feet distance in both the eyes. The refractive correction was $-1.25 \mathrm{DS}$ in both the eyes. Intraocular pressure was $10 \mathrm{mmHg}$ bilaterally. Pupillary light reflex was sluggish bilaterally with no relative afferent pupillary defect (RAPD). Ocular motility was full. Dilated fundal examination showed bilateral hyperemic optic nerve heads (Figure 1). There was no peripapillary telangiectasia. Color vision test using Ishihara chart was reduced to one plate bilaterally. Visual field test showed high false negative, severe general depression and central scotoma. Patient's medical, psychosocial history and family history was unremarkable. Systemic examination was also unremarkable. Blood investigations showed deranged liver function tests but were negative for other tests, including vitamin B12, angiotensin converting enzyme, autoimmune conditions, syphilis and tuberculosis. Diagnosis of toxic optic neuropathy was made and he was started on folate $(150 \mathrm{mcg} /$ day) and thiamine (1.2mg/day) supplementation for 3 months. He was advised to refrain from using gutka or any other form of tobacco. The patient was counselled in detail about the diagnosis and poor visual prognosis. He was also referred to gastroenterologist for deranged LFTs. A follow up 3 months later showed no improvement in the vision and bilateral temporal pallor of both her optic nerve heads. He was not able to perform automated perimetric test.

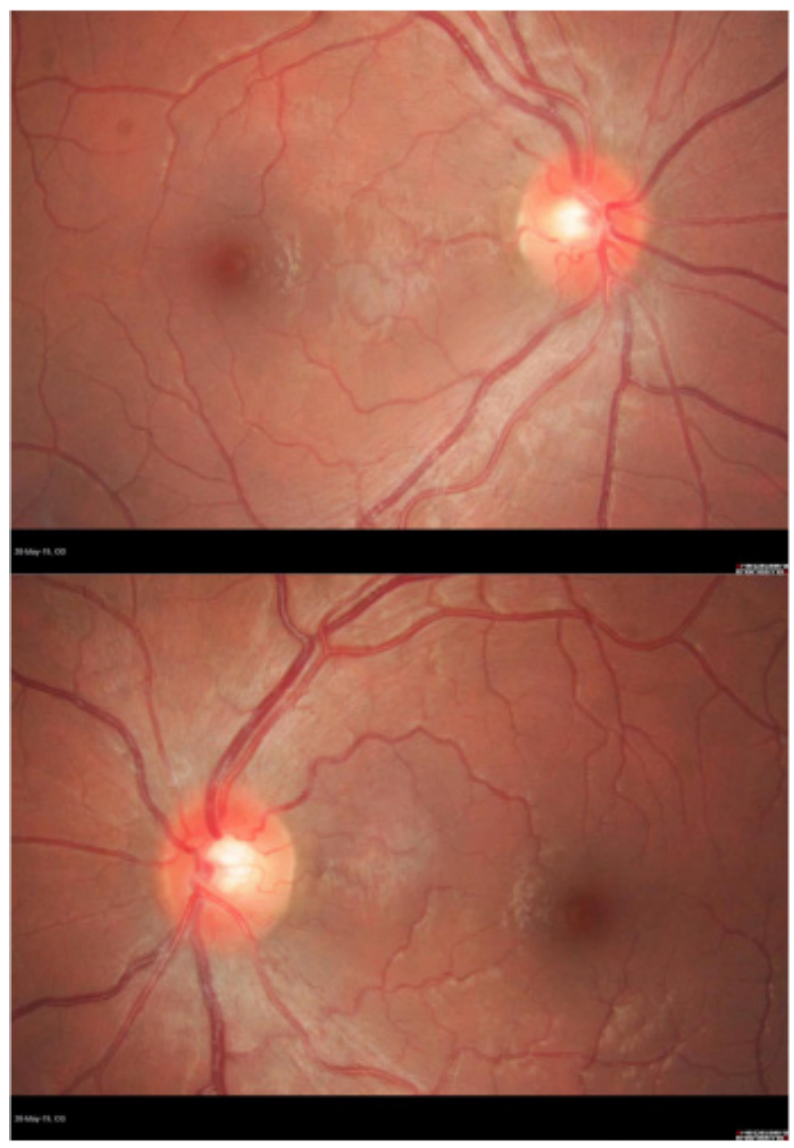

Figure I A (Right) and I b (Left): Fundal examination showing bilateral hyperemic optic nerve heads. 


\section{Discussion}

Gutka consists of finely chopped, sun dried and roasted tobacco mixed with slaked lime, catechu, areca nut and a number of different spices. ${ }^{1}$ Chemical analysis of gutkha showed the presence of polyaromatic hydrocarbons, nitrosamines, and toxic metals such as arsenic, lead, cadmium, nickel, polyaromatic hydrocarbons and residual pesticides, with wide variation between different brands..$^{1,3}$ It is placed between the buccal mucosa and the gums and is either sucked or chewed. Gutka is a form of smokeless tobacco which has gained a high popularity in south Asia among all age groups including children. ${ }^{1-4}$ Though served as a delight, gutka has shown to cause several adverse effects, mainly head and neck cancers. ${ }^{1,3}$ Nevertheless, no association of toxic optic neuropathy with gutka has been recorded.

Toxic optic neuropathy refers to visual impairment due to optic nerve damage by a toxin. ${ }^{5}$ Detailed history, neuroimaging and blood screening for toxins, infection, inflammation and nutritional deficiencies are essential to investigate toxic optic neuropathy. ${ }^{5,6}$ The clinical pattern of this case followed the typical pattern of toxic optic neuropathy. In this case thorough blood wok up was conducted as well as imaging and tests such as fundus photography, perimetry and rereporting of the previous MRI. As the patient presented 1 month after start of symptoms it could be that the imaging and testing was not as accurate as it could have been when the symptoms occurred.

Toxic optic neuropathy usually presents with dyschromatopsia as the first symptom followed by a visual decline that usually is bilateral, symmetrical and painless associated with a central or centrocecal scotoma. Neuroimaging typically yields normal results in toxic optic neuropathy. Cessation of tobacco use can sometimes lead to improvement; however visual deficits often remain long-term. ${ }^{5}$ Generally vitamin B complex supplementation is also prescribed as they act as antioxidants providing protection to the optic nerve against free radical damage. ${ }^{6}$ In this case there was no visual improvement.

The exact mechanism of toxic optic neuropathy is unknown, however, it is hypothesized that the toxin impairs optic nerve's vascular supply or metabolism. Tobacco in gutka may exert its effects by producing free radicals which cause direct damage to the papillomacular bundle in the optic nerve. ${ }^{6}$ Nicotine, with its vasoconstrictive action of nicotine may cause ischemic damage to the optic nerve by reducing its blood flow. ${ }^{6}$ Lead, another chemical found in gutka, has been reported to cause optic neuropathy. ${ }^{7}$ Other toxic chemicals, like arsenic found in gutka can further affect the optic nerve. Furthermore, the unusual pattern of vascular supply of optic nerve head may predispose it to the toxic agent's accumulation.

\section{Conclusion}

To conclude, COVID pandemic carries a risk of increase in substance abuse. ${ }^{8}$ Smokeless tobacco, particularly gutka is on the rise in many parts of the world. ${ }^{1-4}$ This case report aims to inform health professionals to be aware of gutka as a possible cause of toxic optic neuropathy.

\section{Acknowledgments}

None.

\section{Conflicts of interest}

The authors declare no conflicts of interest.

\section{Funding}

None to declare.

\section{Declaration}

Ethics approval was obtained from the institute's Ethical Review Comittee. Consent was obtained from the patient for paticipation and publication of case and fundus pictures.

\section{References}

1. Willis DN, Popovech MA, Gany F, et al. Toxicity of gutkha, a smokeless tobacco product gone global: is there more to the toxicity than nicotine? Int J Environ Res Public Health. 2014;11(1):919-933.

2. Tobacco control : Pakistan country.

3. Niaz K, Maqbool F, Khan F, et al. Smokeless tobacco (paan and gutkha) consumption, prevalence, and contribution to oral cancer. Epidemiol Health. 2017;39:e2017009.

4. Sinha DN, Gupta PC, Kumar A, et al. The Poorest of Poor Suffer the Greatest Burden From Smokeless Tobacco Use: A Study From 140 Countries. Nicotine Tob Res. 2018;20(12):1529-1532.

5. Sharma P, Sharma R. Toxic Optic Neuropathy. Indian J Ophthalmol. 2011;59(2):137-141.

6. Grzybowski A, Holder GE. Tobacco Optic Neuropathy (TON) - The Historical and Present Concept of the Disease. Acta Ophthalmol. 2011;89(5):495-499.

7. Kaveh Abri Aghdam, Amin Zand, Mostafa Soltan Sanjari, et al. Bilateral Optic Disc Edema in a Patient with Lead Poisoning. J Ophthalmic Vis Res. 2019;14(4):513-517.

8. Sun Y, Li Y, Bao Y, et al. Brief Report: Increased Addictive Internet and Substance Use Behavior During the COVID-19 Pandemic in China. The American Journal on Addiction. 4 Jun. 2020. 